\title{
Viva Podestà! Wie humanitäre Interventionen legitimiert und ihr Missbrauch verhindert werden könnte
}

\author{
Gerd Grözinger*
}

$\mathrm{D}$ er Vergleich Libyen-Syrien zeigt: Es dürfte nun eindeutig sein, dass nicht der Umfang des Leids darüber entscheidet, ob die internationale Gemeinschaft interveniert. Dabei scheinen beide Fälle zunächst große Ähnlichkeit zu haben: zwei arabische Länder, beide seit Langem Diktaturen, jeweils mit tiefen internen Spannungen, ein (auch) bewaffneter Widerstand entwickelt sich, und die Arabische Liga wendet sich zunehmend von den Regimen ab. Aber: Im Falle Syriens vermuten die Vereinten Nationen (VN) Anfang 2012 mehr als 5.000 Tote infolge innerstaatlicher Auseinandersetzungen - und nichts passiert. Anders in Libyen. Hier verständigte sich der VN-Sicherheitsrat im März 2011 auf die Resolution 1973, mit der die Errichtung einer Flugverbotszone über Libyen beschlossen wurde. Gleichzeitig nutzten die USA, Frankreich, Großbritannien und ihre militärischen Mitstreiter das Flugverbot exzessiv aus, ja überdehnten es geradezu, um im Bürgerkrieg zu intervenieren. Dieser „Trick“ wird ihnen von den dadurch düpierten anderen Vetostaaten im Sicherheitsrat im Falle Syriens nun zum Verhängnis. Es droht eine völlige Blockade, nicht einmal ein Waffenembargo scheint mehr durchsetzbar zu sein.

Aber vielleicht ist diese Art von internationalem Krisenmanagement unter dem Schutz und der Fahne des VN-Sicherheitsrats generell etwas veraltet und bleibt hinter dem heute Möglichen zurück. Wir wissen, dass ein Großteil der Staaten dieser Welt Diktaturen sind, und auch, dass die Mitgliedschaft als Ständiges Mitglied im Sicherheitsrat mit Vetorecht nicht einer vorbildlich rechtsstaatlich-demokratischen Verfasstheit, sondern nur der puren militärischen Bedeutung dieser Staaten zu verdanken ist. So lange es die Hauptaufgabe der VN-Gremien war, die Eskalation des Kalten Kriegs zu einer alles verschlingenden Apokalypse zu verhindern, war dieser Umstand wohl hinnehmbar, weil alternativlos. Können aber heute, nach Auflösung der Blockstrukturen, nicht andere Verfahren der Legitimitätsprüfung eines kriegerischen Aktes aus humanitären Gründen gefunden werden, jenseits einer Genehmigung des Sicherheitsrats?

$\mathrm{Zu}$ einfach darf man es sich damit nicht machen. Für manche Kommentatoren, besonders von Seiten französischer Intellektueller, sind schon Videos aufständischer Demonstranten in Bengasi, Homs oder anderswo ausreichender Grund, ein Eingreifen für gerechtfertigt, sogar für geboten zu halten. Reine Medienberichte können aber den Mangel einer Sicherheitsratsresolution nicht kompensieren. Die Welt würde eine sehr unsichere, wenn

* Prof. Dr. Gerd Grözinger lehrt Sozial- und Bildungsökonomie an der Universität Flensburg.
Momentaufnahmen jubelnder Menschen, glücklich, gerade einem diktatorischen oder vielleicht auch nur in dieser Region ungeliebten Regime entkommen zu sein, künftig schon als ausreichender Grund für eine Intervention - von welcher Seite auch immer - angesehen würde.

Man kann aber sehr wohl fragen, ob nicht eine eindeutige nachträgliche Billigung einer Intervention durch die Bevölkerung eines solchen Landes die fehlende Vorab-Legitimität kompensieren könnte. Im konkreten Fall wäre das dann der Fall, wenn in Syrien Bodentruppen der NATO, der Arabischen Liga, der EU oder welcher Koalition auch immer das Assad-Regime bezwängen und dann in angemessener Frist nach dem gewonnenen Krieg eine Abstimmung stattfände. Und wenn dabei von der Bevölkerung die Frage zu entscheiden wäre, ob ihrer Meinung nach der Nutzen des militärischen Eingreifens durch den Zugewinn an größerer Freiheit und Sicherheit seine Kosten an Tod und Zerstörung übersteigt. Bei einer Bejahung könnte die von ausländischen Truppen durchgeführte politische Veränderung völkerrechtlich als akzeptables Substitut für einen innenpolitischen Regimewechsel angesehen werden, der ja bei Diktaturen oft ebenfalls mit kriegerischen Handlungen verbunden ist.

Entlehnt ist diese Überlegung der italienischen Geschichte, wo sich im späten Mittelalter die oft bis auf den Tod verfeindeten Adelsgeschlechter eines Stadtstaates gerne einen Herrscher samt Administration von außen holten und diese auf Zeit bestellten. Am Ende ihrer Amtsperiode mussten solche Podestà Rechenschaft über ihre Amtsführung ablegen. Ließen sie sich etwas zuschulden kommen, wurde das vereinbarte Entgelt gekürzt. In einer Bürgerkriegssituation mit einer an der Macht klebenden Diktatur-Elite ist eine solche Lösung a priori nicht möglich. Aber eine nachträgliche Überprüfung der Fremdbefreiung durch ein Referendum kann als angemessenes Substitut dafür gelten.

Natürlich wirft ein solches Verfahren viele mögliche Detailfragen auf. Vor allem: Ist nicht die Gesamtbevölkerung des angegriffenen Landes eine zu große Gruppe, als dass man sie dazu befragen könnte? Sollten nicht nur die direkt Betroffenen, also die Verwundeten, Ausgebombten, die Familienangehörigen der Getöteten, allein abstimmen? Das ist aus zwei Gründen wenig angemessen. Erstens wäre es falsch, nur Angehörige des Militärs zu befragen. Zwar kommt es in Gewaltkonflikten in ihren Reihen üblicherweise zu besonders hohen Opferzahlen, doch sind sie häufig Nutznießer des alten Regimes gewesen. Diesen auch dann noch die Rolle von Sprechern für eine Bevölkerung insgesamt einzuräumen, wenn die Diktatur zu einem Ende kommt, der sie gerade noch gedient haben, erscheint widersinnig. Zweitens muss wieder auf die Intervention als Alternative zu einer inneren Erhebung verwiesen werden. Auch ein Auf- 
stand ist in der Regel opferreich, meist sogar in viel stärkerem Maße als im Fall des Einmarschs einer High-Tech-Armee. Und trotzdem würde die Weltgemeinschaft vermutlich ein so herbeigeführtes Ende einer Diktatur für legitim erachten, wenn dies denn von der Mehrheit der jeweiligen Bevölkerung des betroffenen Landes getragen würde. Deshalb ist eine ordnungsgemäß durchgeführte Abstimmung innerhalb der ganzen Bevölkerung notwendig.

Und hier kämen als neutrale Bestätigungsinstanz der Ordnungsmäßigkeit eines solchen Referendums wohl nur die VN in Frage. Trotz aller Kritik an den Vereinten Nationen: keine andere Einrichtung besitzt einen ähnlichen Neutralitätsbonus. Wie könnte ein solcher Prozess praktisch aussehen? Die VN hätten zuerst festzustellen, dass ein „noch nicht legitimer“ Krieg stattgefunden hat, der aber nachträglich legitimiert werden könnte. Sie hätte dann dafür eine Task-Force zur Beobachtung und Begleitung dieses Prozesses einzusetzen. Einen solchen Beschluss könnte, jeweils auf Antrag eines Mitgliedslandes, jede der drei großen VN-Einrichtungen treffen: entweder der Sicherheitsrat, die Generalversammlung oder auch der Internationale Gerichtshof.

Die Task-Force der VN wäre dann auch für den ordnungsgemäßen Ablauf der Volksabstimmung zuständig. In einem engen Zeitrahmen, zu denken ist an zwei bis fünf Jahre, könnte sie einen Termin für ein Referendum ansetzen. Innerhalb dieses Zeitfensters sollte es möglich sein, ausreichend stabile Verhältnisse wiederherzustellen. Die Vereinten Nationen müssten zudem Wahlgebiet und Wahlberechtigte bestimmen. Dies könnte sich als schwierige Aufgabe erweisen. Dürfte etwa im Falle Kosovo nur die eigene Bevölkerung abstimmen oder könnten auch Menschen aus dem bombardierten Serbien teilnehmen? Die VN müsste in einem solchen uneindeutigen Fall früh Zugang zu den Einwohnerlisten haben. Und sie hätte für Informationsfreiheit zu sorgen, um auch schon vor dem Abstimmungstermin die nötigen Bedingungen für eine vernünftige und unabhängige Meinungsbildung zu schaffen.

Der Zwang, sich eines solchen Verfahrens nachträglicher Legitimierung zu unterziehen, würde den angreifenden Ländern von Anfang an nützliche faktische Restriktionen auferlegen. Um die spätere Abstimmung nicht zu verlieren, würden sie versuchen, die Zahl der Opfer im Land gering zu halten und besonders die Zivilbevölkerung zu schützen. Wegen der längeren Zeitspanne vom Einmarsch bis zum Urnengang müssten sie zusätzlich dafür sorgen, dass die politische Nachkriegsordnung breit akzeptiert wird. Sie könnten sich allzu ungerechtfertigte Verträge, z.B. zum Zwecke der Rohstoffausbeutung, nicht leisten, weil auch dies die Meinung der zu Befragenden negativ beeinflusst. Und sie müssten vor allem ökonomische Ressourcen aufwenden, um die Lebensbedingungen insgesamt zu verbessern. Hätte die internationale Koalition in Afghanistan gewusst, dass sie sich in wenigen Jahren dort einem Referendum zu unterwerfen haben, wären von Anfang sicher sehr viel mehr Mittel für den zivilen Aufbau geflossen. Und es wäre, aus Angst vor den Folgen bei der Volksabstimmung, vielleicht auch die militärisch scheinbar so kostengünstige Lösung unterblieben, sich korrupter und ihrerseits mörderischer Hilfstruppen zu bedienen.
Und was sollte passieren, wenn eine Interventionsmacht kein Referendum zulässt? Oder wenn die Abstimmung verloren geht, wenn sich also eine Mehrheit im Nachhinein gegen die Intervention ausspricht? Dann wären gegen die Interventen von der neutralen Task-Force der VN Strafen zu verhängen. Z.B. könnten die politischen Eliten, die den Krieg als Regierungsmitglieder, als Abgeordnete, als höhere Funktionäre zu verantworten haben, mit einem politischen Funktionsverbot belegt werden. Das wäre in den Gesetzen aller Mitgliedstaaten auf Verlangen der VN vorab zu verankern. Vermutlich würden deren Machteliten bei der Planung militärischer Aktionen sehr viel vorsichtiger werden. Es könnten auch Verträge mit Firmen des Angreiferlandes oder solche über Militärbasen und Militärbündnisse als automatisch gekündigt gelten; ihre erneute Verhandlung unterläge in diesem Fall einer im Abstimmungsland baldigst neu zu wählenden Regierung. Man könnte auch an empfindliche Geldbußen denken. Diese dürften aber nicht direkt an den betroffenen Staat fließen, um nicht durch Eigennutz erzeugtes Abstimmungsverhalten zu provozieren, aber durchaus an mit Kriegsverhinderung, der Versorgung von Flüchtlingen und dem Aufbau von Zivilgesellschaften befassten Programmen der VN. Oder alternativ: Mitgliedstaaten könnte es erlaubt werden, Extrazölle auf Importe aus den intervenierenden Staaten zu erheben, mit der Verpflichtung, diese zu einem großen Anteil an die VN abzuführen.

Die Europäische Union sollte darüber nachdenken, ob sie nicht diplomatische Vorstöße in die hier skizzierte Richtung einer nachträglichen Legitimierung kriegerischer Aktivitäten aus humanitären Gründen für angebracht hält. Sicher wäre eine Weltgemeinschaft mit ausschließlich demokratisch-rechtstaatlich verfassten Mitgliedern vorzuziehen. In diesem Fall wären solche Überlegungen gar nicht nötig. Aber in der gegenwärtigen Situation wäre eine Pflicht zur nachträglichen Überprüfung durch ein Referendum in angegriffenen Staaten doch eine erhebliche Verbesserung gegenüber einem Jetztzustand, in dem Befreiungsbewegungen entweder allein gelassen werden oder zum Spielball machtpolitischer Interessen verkommen. 